\title{
Commuting Structure Jacobi Operator for Real Hypersurfaces in Complex Space Forms
}

\author{
U-Hang $\mathrm{Ki}^{1}$, Hiroyuki Kurihara ${ }^{2}$ \\ ${ }^{1}$ The National Academy of Science, Seoul, Korea \\ ${ }^{2}$ Hachinohe National College of Technology, Aomori, Japan \\ Email: uhangki2005@yahoo.co.kr, kurihara-g@hachinohe-ct.ac.jp
}

Received October 9, 2012; revised December 7, 2012; accepted December 20, 2012

\begin{abstract}
Let $M$ be a real hypersurface of a complex space form with almost contact metric structure $(\phi, \xi, \eta, g)$. In this paper, we prove that if the structure Jacobi operator $R_{\xi}=R(\cdot, \xi) \xi$ is $\phi \nabla_{\xi} \xi$-parallel and $R_{\xi}$ commute with the shape operator, then $M$ is a Hopf hypersurface. Further, if $R_{\xi}$ is $\phi \nabla_{\xi} \xi$-parallel and $R_{\xi}$ commute with the Ricci tensor, then $M$ is also a Hopf hypersurface provided that $\operatorname{Tr} R_{\xi}$ is constant.
\end{abstract}

Keywords: Complex Space Form; Hopf Hypersurface; Structure Jacobi Operator; Shape Operator; Ricci Tensor

\section{Introduction}

A complex $n$-dimensional Kähler manifold of constant holomorphic sectional curvature $4 c \neq 0$ is called a complex space form, which is denoted by $M_{n}(c)$. So naturally there exists a Kähler structure $J$ and Kähler metric $g$ on $M_{n}(c)$. Now let us consider a real hypersurface $M$ in $M_{n}(c)$. Then we also denote by $g$ the induced Riemannian metric of $M$ and by $N$ a local unit normal vector field of $M$ in $M_{n}(c)$. Further, $A$ denotes by the shape operator of $M$ in $M_{n}(c)$. Then, an almost contact metric structure $(\phi, \xi, \eta, g)$ of $M$ is naturally induced from the Kähler structure of $M$ as follows:

$$
\phi X=(J X)^{\mathrm{T}}, \xi=-J N, \eta(X)=g(X, \xi), X \in T M,
$$

where $T M$ denotes the tangent bundle of $M$ and ()$^{\mathrm{T}}$ the tangential component of a vector. The Reeb vector $\xi$ is said to be principal if $A \xi=\alpha \xi$, where $\alpha=\eta(A \xi)$. A real hypersurface is said to a Hopf hypersurface if the Reeb vector $\xi$ of $M$ is principal. Hopf hypersurfaces is realized as tubes over certain submanifolds in $P_{n} \mathbb{C}$, by using its focal map (see Cecil and Ryan [1]). By making use of those results and the mentioned work of Takagi [2,3], Kimura [4] proved the local classification theorem for Hopf hypersurfaces of $P_{n} \mathbb{C}$ whose all principal curvatures are constant. For the case $H_{n} \mathbb{C}$, Berndt [5] proved the classification theorem for Hopf hypersurfaces whose all principal curvatures are constant. Among the several types of real hypersurfaces appeared in Takagi's list or Berndt's list.
The Reeb vector field $\xi$ plays an important role in the theory of real hypersurfaces in a complex space form $M_{n}(c)$. Related to the Reeb vector field $\xi$ the Jacobi operator $R_{\xi}$ defined by $R_{\xi}=R(\cdot, \xi) \xi$ for the curvature tensor $R$ on a real hypersurface $M$ in $M_{n}(c)$ is said to be a structure Jacobi operator on $M$. The structure Jacobi operator has a fundamental role in contact geometry. In [6], Cho and first author started the study on real hypersurfaces in complex space form by using the operator $R_{\xi}$. In particular the structure Jacobi operator has been studied under the various commutative condition [7-9]. For example, Pérez et al. [9] called that real hypersurfaces $M$ has commuting structure Jacobi operator if $R_{\xi} R_{X}=R_{X} R_{\xi}$ for any vector field $X$ on $M$, and proved that there exist no real hypersurfaces in $M_{n}(c)$ with commuting structure Jacobi operator. On the other hand Ortega et al. [10] have proved that there are no real hypersurfaces in $M_{n}(c)$ with parallel structure Jacobi operator $R_{\xi}$, that is, $\nabla_{X} R_{\xi}=0$ for any vector field $X$ on $M$. More generally, such a result has been extended by [11]. In this situation, if naturally leads us to be consider another condition weaker than parallelness. In the preceding work, we investigate the weaker condition $\xi$-parallelness, that is, $\nabla_{\xi} R_{\xi}=0$ (cf. $[8,12,13])$.

In this paper we consider the notion of $\phi \nabla_{\xi} \xi$-parallel structure Jacobi operator $R_{\xi}$, that is, $\nabla_{\phi \nabla_{\xi} \xi} R_{\xi}=0$ for the vector $\phi \nabla_{\xi} \xi$ orthogonal to $\xi$. Further we invetigate the structure Jacobi operator is $\phi \nabla_{\xi} \xi$-parallel under the condition that the structure Jacobi operator commute with the shape operator or the Ricci tensor. 
This paper consists of two parts. In the first part of this paper, we prove that if the structure Jacobi operator $R_{\xi}=R(\cdot, \xi) \xi$ is $\phi \nabla_{\xi} \xi$-parallel and $R_{\xi}$ commute with the shape operator, then $M$ is a Hopf hypersurface (see Theorem 1 in Section 4). In the second part of this paper, we prove that if $R_{\xi}$ is $\phi \nabla_{\xi} \xi$-parallel and $R_{\xi}$ commute with the Ricci tensor, then $M$ is also a Hopf hypersurface provided that $\operatorname{Tr} R_{\xi}$ is constant (see Theorem 2 in Section 5).

All manifolds in this paper are assumed to be connected and of class $C^{\infty}$ and the real hypersurfaces are supposed to be oriented.

\section{Fundamental Facts of Real Hypersurface}

In this section the elemental factors of a real hypersurface are recalled. Let $M$ be a real hypersurface in a complex space form $M_{n}(c)$ with constant holomorphic sectional curvature $4 c, c \neq 0$ and $N$ be a unit normal vector field on $M$. By $\tilde{\nabla}$ we denote the Levi-Civita connection with respect to the Fubini-Study metric $\tilde{g}$ of $M_{n}(c)$. Then the Gauss and Weingarten formulas are respectively given by

$$
\tilde{\nabla}_{X} Y=\nabla_{X} Y+g(A X, Y) N, \tilde{\nabla}_{X} N=-A X
$$

for any vector fields $X$ and $Y$ on $M$, where $g$ denotes the Riemannian metric of $M$ induced from $\tilde{g}$ and $A$ is the shape operator of $M$ in $M_{n}(c)$. For any vector field $X$ tangent to $M$, we put

$$
J X=\phi X+\eta(X) N, J N=-\xi,
$$

for the complex structure $J$ of $M_{n}(c)$. We call $\xi$ the Reeb vector field. Then we may see that the aggregate $(\phi, \xi, \eta, g)$ is an almost contact metric structure on $M$, that is, we have

$$
\begin{aligned}
& \phi^{2} X=-X+\eta(X) \xi, \\
& g(\phi X, \phi Y)=g(X, Y)-\eta(X) \eta(Y), \\
& \eta(\xi)=1, \phi \xi=0, \eta(X)=g(X, \xi)
\end{aligned}
$$

for any vector fields $X$ and $Y$ on $M$. From Kähler condition $\tilde{\nabla} J=0$, and making use of Gauss and Weingarten formulas, we obtain

$$
\left(\nabla_{X} \phi\right) Y=\eta(Y) A X-g(A X, Y) \xi
$$

and

$$
\nabla_{X} \xi=\phi A X
$$

for any vector fields $X, Y$ tangent to $M$.

The equations of Gauss and Codazzi are respectively given by the following:

$$
\begin{aligned}
R & (X, Y) Z \\
= & c(g(Y, Z) X-g(X, Z) Y+g(\phi Y, Z) \phi X \\
& -g(\phi X, Z) \phi Y-2 g(\phi X, Y) \phi Z) \\
& +g(A Y, Z) A X-g(A X, Z) A Y
\end{aligned}
$$

and

$$
\begin{aligned}
& \left(\nabla_{X} A\right) Y-\left(\nabla_{Y} A\right) X \\
& =c(\eta(X) \phi Y-\eta(Y) \phi X-2 g(\phi X, Y) \xi),
\end{aligned}
$$

where $R$ denotes the curvature tensor of $M$.

In what follows, to write our formulas in convention forms, we denote by

$\alpha=\eta(A \xi), \beta=\eta\left(A^{2} \xi\right), \gamma=\eta\left(A^{3} \xi\right)$ and $h=\operatorname{Tr} A$, and for a function $f$ we denote by $\nabla f$ the gradient vector field of $f$.

If we put $U=\nabla_{\xi} \xi$, then $U$ is orthogonal to the Reeb vector field $\xi$. We get

$$
\phi U=-A \xi+\alpha \xi,
$$

which shows that $g(U, U)=\beta-\alpha^{2}$. Thus we easily verify that $\xi$ is a principal curvature vector, that is $A \xi=\alpha \xi$ if and only if $\beta-\alpha^{2}=0$.

From Gauss Equation (2.3), the Ricci tensor $S$ of $M$ is given by

$$
S X=c\{(2 n+1) X-3 \eta(X) \xi\}+h A X-A^{2} X
$$

for any vector field $X$ on $M$.

If $A \xi-g(A \xi, \xi) \xi \neq 0$, then we can put

$$
A \xi=\alpha \xi+\mu W,
$$

where $W$ is a unit vector field orthogonal to $\xi$. Then by (2.2) we see that $U=\mu \phi W$ and hence $g(U, U)=\mu^{2}$. So we have

$$
\mu^{2}=\beta-\alpha^{2} .
$$

In this paper, we basically use the technical computations with the orthogonal triplet $\{\xi, U, W\}$ and their associated scalars $\alpha, \beta$ and $\mu$.

Using (2.2) and (2.7), it is seen that

$$
\begin{gathered}
\mu g\left(\nabla_{X} W, \xi\right)=g(A U, X), \\
g\left(\nabla_{X} \xi, U\right)=\mu g(A W, X) .
\end{gathered}
$$

Now, differentiating (2.5) covariantly along $M$ and making use of (2.1), (2.2) and (2.4), we find

$$
\begin{gathered}
\left(\nabla_{X} A\right) \xi=-\phi \nabla_{X} U+g(A U+\nabla \alpha, X) \xi \\
-A \phi A X+\alpha \phi A X,
\end{gathered}
$$

which enables us to obtain

$$
\left(\nabla_{\xi} A\right) \xi=2 A U+\nabla \alpha .
$$

By the definition of $U,(2.2)$ and (2.12), it is verified 
that

$$
\nabla_{\xi} U=3 \phi A U+\alpha A \xi-\beta \xi+\phi \nabla \alpha .
$$

From the Gauss Equation (2.3) the structure Jacobi operator $R_{\xi}$ is given by

$$
\begin{aligned}
& R_{\xi}(X)=R(X, \xi) \xi \\
& =c\{X-\eta(X) \xi\}+\alpha A X-\eta(A X) A \xi
\end{aligned}
$$

for any vector field $X$ on $M$.

Let $\Omega$ be the open subset of $M$ defined by

$$
\Omega=\{p \in M ; \mu(p) \neq 0\} .
$$

At each point of $\Omega$, the Reeb vector field $\xi$ is not principal. That is, $\xi$ is not an eigenvector of the shape operator $A$ of $M$ if $\Omega \neq \varnothing$.

In what follows we assume that $\Omega$ is not an empty set in order to prove our main theorem by reductio ad absurdum, unless otherwise stated, all discussion concerns the set $\Omega$.

\section{Real Hypersurfaces Satisfying $\boldsymbol{R}_{\xi} S=S R_{\xi}$}

Let $M$ be a real hypersurface in $M_{n}(c), c \neq 0$ satisfying $R_{\xi} S=S R_{\xi}$, which means that the Ricci tensor $S$ of type $(1,1)$ and the structure Jacobi operator $R_{\xi}$ commute to each other. Then by (2.6) and (2.14) we have

$$
\begin{aligned}
g & \left(R_{\xi}(Y), S X\right)-g\left(R_{\xi}(X), S Y\right) \\
= & g\left(A^{3} \xi, Y\right) g(A \xi, X)-g\left(A^{3} \xi, X\right) g(A \xi, Y) \\
& -g\left(A^{2} \xi, Y\right) g(h A \xi-c \xi, X) \\
& +g\left(A^{2} \xi, X\right) g(h A \xi-c \xi, Y) \\
& -c h(g(A \xi, Y) \eta(X)-g(A \xi, X) \eta(Y)),
\end{aligned}
$$

which shows that

$$
\alpha A^{3} \xi=(\alpha h-c) A^{2} \xi+(\gamma-\beta h+c h) A \xi+c(\beta-h \alpha) \xi .
$$

Combining above two equations and using (2.7), we obtain

$$
\begin{aligned}
& \mu\left(g\left(A^{2} \xi, Y\right) w(X)-g\left(A^{2} \xi, X\right) w(Y)\right) \\
& =\beta(\eta(Y) g(A \xi, X)-\eta(X) g(A \xi, Y)),
\end{aligned}
$$

where a 1-form $w$ is defined by $w(X)=g(W, X)$ for any vector field $X$. Putting $Y=A \xi$ in this, we find

$$
\mu^{2} g\left(A^{2} \xi, X\right)=\mu \gamma w(X)-\beta \alpha g(A \xi, X)+\beta^{2} \eta(X),
$$

which shows that

$$
\mu^{2} A^{2} \xi=(\gamma-\beta \alpha) A \xi+\left(\beta^{2}-\alpha \gamma\right) \xi .
$$

Comparing (3.1) with (3.2), we find

$$
(h-\rho)(\beta-\rho \alpha-c)=0
$$

on $\Omega$, where we have put $\mu^{2} \rho=\gamma-\beta \alpha$ and $\mu^{2}(\beta-\rho \alpha)=\beta^{2}-\alpha \gamma$. So (3.2) becomes on $\Omega$

$$
A^{2} \xi=\rho A \xi+(\beta-\rho \alpha) \xi,
$$

which together with (2.7) yields

$$
A W=\mu \xi+(\rho-\alpha) W
$$

and hence

$$
A^{2} W=\rho A W+(\beta-\rho \alpha) W .
$$

Now, differentiating (3.5) covariantly along $\Omega$, we find

$$
\begin{aligned}
& \left(\nabla_{X} A\right) W+A \nabla_{X} W \\
& =(X \mu) \xi+\mu \nabla_{X} \xi+X(\rho-\alpha) W+(\rho-\alpha) \nabla_{X} W .
\end{aligned}
$$

By taking the inner product with $W$ in the last equation, we obtain

$$
g\left(\left(\nabla_{X} A\right) W, W\right)=-2 g(A U, X)+X \rho-X \alpha
$$

since $W$ is a unit vector field orthogonal to $\xi$. We also have by applying $\xi$ to (3.7) and making use of (2.9)

$$
\mu g\left(\left(\nabla_{X} A\right) W, \xi\right)=(\rho-2 \alpha) g(A U, X)+\mu(X \mu),
$$

which together with the Codazzi Equation (2.4) gives

$$
\begin{gathered}
\mu\left(\nabla_{W} A\right) \xi=(\rho-2 \alpha) A U-2 c U+\mu \nabla \mu, \\
\mu\left(\nabla_{\xi} A\right) W=(\rho-2 \alpha) A U-c U+\mu \nabla \mu .
\end{gathered}
$$

Putting $X=\xi$ in (3.8) and using (3.11), we obtain

$$
W \mu=\xi \rho-\xi \alpha=: \xi \lambda,
$$

where we have put $\lambda=w(A W)$. Differentiating (3.4) covariantly and using (2.2) we find

$$
\begin{aligned}
& g\left(\left(\nabla_{X} A\right) A \xi, Y\right)+g\left(A\left(\nabla_{X} A\right) \xi, Y\right) \\
& +g\left(A^{2} \phi A X, Y\right)-\rho g(A \phi A X, Y) \\
& =(X \rho) g(A \xi, Y)+\rho g\left(\left(\nabla_{X} A\right) \xi, Y\right) \\
& +X(\beta-\rho \alpha) \eta(Y)+(\beta-\rho \alpha) g(\phi A X, Y),
\end{aligned}
$$

which together with (2.4) and (2.12) implies that

$$
\left(\nabla_{\xi} A\right) A \xi=\rho A U-c U+\frac{1}{2} \nabla \beta .
$$

If we replace $X$ by $\xi$ in (3.13) and make use of (2.4), (2.12) and the last equation, then we get

$$
\begin{aligned}
& 3 A^{2} U-2 \rho A U+(\alpha \rho-\beta-c) U \\
& =(\xi \rho) A \xi+\xi(\beta-\alpha \rho) \xi-A \nabla \alpha+\rho \nabla \alpha-\frac{1}{2} \nabla \beta .
\end{aligned}
$$

Now, we define a 1 -form $u$ by $u(X)=g(U, X)$ 
for any vector field $X$, it is, using (2.4) and (3.13), seen that

$$
\begin{aligned}
c & (u(Y) \eta(X)-u(X) \eta(Y))+2 c(\rho-\alpha) g(\phi Y, X) \\
& -g\left(A^{2} \phi A X, Y\right)+g\left(A^{2} \phi A Y, X\right) \\
& +2 \rho g(\phi A X, A Y) \\
& -(\beta-\rho \alpha)(g(\phi A Y, X)-g(\phi A X, Y)) \\
= & g\left(A Y,\left(\nabla_{X} A\right) \xi\right)-g\left(A X,\left(\nabla_{Y} A\right) \xi\right) \\
\quad & +(Y \rho) g(A \xi, X)-(X \rho) g(A \xi, Y) \\
& +Y(\beta-\rho \alpha) \eta(X)-X(\beta-\rho \alpha) \eta(Y)
\end{aligned}
$$

If replace $X$ by $\mu W$ to both sides of (3.15) and take account of (2.12), (3.5), (3.6), (3.9) and (3.10), then we obtain

$$
\begin{aligned}
& (3 \alpha-2 \rho) A^{2} U+2\left(\rho^{2}+\beta-2 \rho \alpha+c\right) A U \\
& +(\rho-\alpha)(\beta-\rho \alpha-2 c) U \\
& =\mu A \nabla \mu+(\rho \alpha-\beta) \nabla \alpha-\frac{1}{2}(\rho-\alpha) \nabla \beta \\
& \quad+\mu^{2} \nabla \rho-\mu(W \rho) A \xi-\mu W(\beta-\rho \alpha) \xi .
\end{aligned}
$$

Differentiating (2.14) covariantly along $\Omega$, we find

$$
\begin{aligned}
g & \left(\left(\nabla_{X} R_{\xi}\right) Y, Z\right) \\
= & g\left(\nabla_{X}\left(R_{\xi} Y\right)-R_{\xi}\left(\nabla_{X} Y\right), Z\right) \\
= & -c\left(\eta(Z) g\left(\nabla_{X} \xi, Y\right)+\eta(Y) g\left(\nabla_{X} \xi, Z\right)\right) \\
& +(X \alpha) g(A Y, Z)+\alpha g\left(\left(\nabla_{X} A\right) Y, Z\right) \\
& -\eta(A Z)\left\{g\left(\left(\nabla_{X} A\right) \xi, Y\right)+g(A \phi A X, Y)\right\} \\
& -\eta(A Y)\left\{g\left(\left(\nabla_{X} A\right) \xi, Z\right)+g(A \phi A X, Z)\right\} .
\end{aligned}
$$

In the following we assume that $M$ satisfies

$\nabla_{\phi \nabla_{\xi} \xi} R_{\xi}=0$. Then we have $\nabla_{W} R_{\xi}=0$ on $\Omega$ because of (2.5) and (2.7). Putting $X=W$ in the last equation and using (2.2), we have

$$
\begin{aligned}
& -c(\eta(Z) g(\phi A W, Y)+\eta(Y) g(\phi A W, Z)) \\
& +(W \alpha) g(A Y, Z)+\alpha g\left(\left(\nabla_{W} A\right) Y, Z\right) \\
& -\eta(A Z)\left(g\left(\left(\nabla_{W} A\right) \xi, Y\right)+g(A \phi A W, Y)\right) \\
& -\eta(A Y)\left(g\left(\left(\nabla_{W} A\right) \xi, Z\right)+g(A \phi A W, Z)\right)=0
\end{aligned}
$$

because of $\nabla_{W} R_{\xi}=0$. If we replace $Y$ by $\xi$ and make use of (2.12) and (3.5), then we obtain

$$
\alpha A \phi A W+c \phi A W=0 \text {. }
$$

Remark 1. $\alpha \neq 0$ on $\Omega$.

If not, then we have $\alpha=0$, and then we restrict our arguments on such a place. From (3.18) we have $\phi A W=0$, which together with (3.5) yields $\rho=0$ and hence (3.5) reformed as $A W=\mu \xi$. But, it is, using (2.8) and (3.12), that $W \beta=0$. So (3.16) turns out to be

$$
2(\beta+c) A U=\frac{1}{2} A \nabla \beta,
$$

where we have used (2.8) and $\rho=\alpha=0$.

On the other hand (3.17) is reduced to

$$
\eta(A Y) g\left(\left(\nabla_{W} A\right) \xi, Z\right)+\eta(A Z) g\left(\left(\nabla_{W} A\right) \xi, Y\right)=0
$$

because of (3.18) with $\alpha=0$. If we replace $Y$ by $W$ and take account of (2.7), (2.8) and (3.10), then we obtain $\left(\nabla_{W} A\right) \xi=0$. Thus (3.10) becomes $\mu \nabla \mu=2 c U$ and consequently $(1 / 2) \nabla \beta=2 c U$ and hence $\xi \beta=0$. Accordingly (3.19) reformed as $\beta A U=0$ and thus $A U=0$. Using these facts, (3.14) is reduced to $(1 / 2) \nabla \beta=(\beta+c) U$. This contradicts the fact that $\nabla \beta=4 c U$. Therefore $\alpha \neq 0$ on $\Omega$ is proved.

If we make use of (3.18) and Remark 1, then (3.17) reformed as

$$
\begin{aligned}
\alpha & \left(\nabla_{W} A\right) X \\
= & -(W \alpha) A X+g(A \xi, X)\left(\nabla_{W} A\right) \xi \\
& +g\left(\left(\nabla_{W} A\right) \xi, X\right) A \xi \\
& -\frac{c}{\alpha} \mu(w(X) \phi A W+g(\phi A W, X) W) .
\end{aligned}
$$

Using (3.5) and (3.10), we can write the last equation as

$$
\begin{aligned}
\alpha & \left(\nabla_{W} A\right) X \\
= & -(W \alpha) A X-\frac{c}{\alpha} \lambda(w(X) U+u(X) W) \\
& +\frac{1}{\mu}\{(\rho-2 \alpha) A U-2 c U+\mu \nabla \mu\} g(A \xi, X) \\
& +\frac{1}{\mu} g((\rho-2 \alpha) A U-2 c U+\mu \nabla \mu, X) A \xi .
\end{aligned}
$$

If we put $X=W$ in (3.20) and make use of (2.8), (3.8) and (3.12), then we obtain

$$
\begin{aligned}
& \frac{1}{2} \nabla \beta-\alpha \nabla \rho \\
& =c\left(2+\frac{\lambda}{\alpha}\right) U-\rho A U+(W \alpha) A W-(\xi \lambda) A \xi .
\end{aligned}
$$

Taking inner product $W$ to this, and using (3.5) and (3.12), we find

$$
\frac{1}{2} W \beta-\alpha(W \rho)=(\rho-\alpha) W \alpha-\mu(W \mu),
$$

which together with (2.8) implies that

$$
W \beta=\alpha(W \rho)+\rho(W \alpha) .
$$

If we take the inner product $\xi$ to (3.21) and make use of (2.7) and (3.5), then we have $\xi \beta=2 \mu(W \alpha)+2 \alpha(\xi \alpha)$, which connected to (2.8) gives 


$$
\xi \mu=W \alpha .
$$

From (2.8) we have $2 \mu(W \mu)=W \beta-2 \alpha(W \alpha)$, which together with (3.12) and (3.22) yields

$$
\alpha(W \lambda)=2 \mu(\xi \lambda)-\lambda(W \alpha) .
$$

\section{Real Hypersurfaces Satisfying $\nabla_{\phi \nabla_{\xi} \xi} R_{\xi}=0$ and $R_{\xi} A=A R_{\xi}$}

Let $M$ be a real hypersurface in $M_{n}(c), c \neq 0$ satisfying $\nabla_{\phi \nabla_{\xi} \xi} R_{\xi}=0$. We have from (2.14)

$$
\begin{aligned}
& g\left(R_{\xi} Y, A X\right)-g\left(R_{\xi} X, A Y\right) \\
= & g\left(A^{2} \xi, Y\right) g(A \xi, X)-g\left(A^{2} \xi, X\right) g(A \xi, Y) \\
& -c(g(A \xi, Y) \eta(X)-g(A \xi, X) \eta(Y)) .
\end{aligned}
$$

In the following we assume that $R_{\xi} A=A R_{\xi}$. Then we have from above equation

$$
A^{2} \xi=\rho A \xi+c \xi
$$

which shows that

$$
\beta=\rho \alpha+c
$$

Substituting (4.1) into the first equation of section 3 , we find $R_{\xi} S-S R_{\xi}=0$. Thus, all relationships (3.3)(3.22) with $\beta=\rho \alpha+c$ are established on $\Omega$. Combining (3.5) to (3.18), we obtain $\lambda(\alpha A U+c U)=0$. So we have

$$
\alpha A U+c U=0 .
$$

In fact, if not, then we have $\lambda=0$, that is, $\rho=\alpha$. Therefore, (3.5) and (4.2) are reduces respectively to $A W=\mu \xi$ and $\mu^{2}=c$. So (3.16) becomes

$$
\alpha A^{2} U+4 c A U=-\mu(W \alpha) A \xi
$$

on this subset. On the other hand, if we take the inner product $W$ to (3.20) and make use of (3.8) and $\mu^{2}=c$, then we obtain $\alpha A U=c U+\mu(W \alpha) \xi$. Comparing this with last equation, we verify that $U=0$, a contradiction, Therefore (4.3) is established on whole space.

Because of (4.2) and (4.3), we can write (3.21) as

$$
\frac{1}{2}(\alpha \nabla \rho-\rho \nabla \alpha)=-c\left(1+\frac{2 \rho}{\alpha}\right) U+(\xi \lambda) A \xi-(W \alpha) A W,
$$

which shows that

$$
\alpha^{2}(U \rho)-\rho \alpha(U \alpha)=-2 c(2 \rho+\alpha) \mu^{2} .
$$

Using (4.3), we can also write (3.20) as

$$
\begin{aligned}
\alpha & \left(\nabla_{W} A\right) X \\
= & -(W \alpha) A X-\frac{c \lambda}{\alpha}(u(X) W+w(X) U) \\
& +g\left(\nabla \mu-\frac{c \rho}{\alpha \mu} U, X\right) A \xi+g(A \xi, X)\left(\nabla \mu-\frac{c \rho}{\alpha \mu} U\right) .
\end{aligned}
$$

Replacing $X$ by $U$ in this and using (4.3), we find

$$
\alpha\left(\nabla_{W} A\right) U=(U \mu) A \xi+\frac{c}{\alpha}\left((W \alpha) U-\lambda \mu^{2} W-\mu \rho A \xi\right) .
$$

If we take the inner product $U$ to (3.7) and take account of (2.4), (2.10) and (4.3), then we obtain

$$
\begin{aligned}
& (\alpha \lambda+c) g\left(\nabla_{X} W, U\right) \\
& =\alpha g\left(\left(\nabla_{W} A\right) X, U\right)+c \alpha \mu \eta(X)-\alpha \mu^{2} g(A W, X),
\end{aligned}
$$

which together with (2.8), (4.2) and (4.6) yields

$$
\begin{aligned}
& \mu^{2} g\left(\nabla_{X} W, U\right) \\
= & g\left(X,(U \mu) A \xi+\frac{c}{\alpha}\left((W \alpha) U-\lambda \mu^{2} W-\mu \rho A \xi\right)\right. \\
& \left.+c \alpha \mu \xi-\alpha \mu^{2} A W\right) .
\end{aligned}
$$

Putting $X=U$ in this, we have

$$
g\left(\nabla_{U} W, U\right)=\frac{c}{\alpha}(W \alpha) .
$$

Now, applying by $\phi$ in (2.11) and using (2.10), we find

$$
\begin{aligned}
& \phi\left(\nabla_{X} A\right) \xi \\
& =\nabla_{X} U-\mu w(A X) \xi-\phi A \phi A X-\alpha A X+\alpha \eta(A X) \xi .
\end{aligned}
$$

If we put $X=U$ in this and make use of (2.5), (3.5) and (4.3), then we obtain

$$
\nabla_{U} U=\phi\left(\nabla_{U} A\right) \xi+c\left(\frac{\lambda}{\alpha}-1\right) U .
$$

Taking the inner product $U$ to (2.11), we also obtain

$$
\begin{aligned}
& g\left(\nabla_{X} W, U\right) \\
& =\frac{1}{\mu} g\left(\left(\nabla_{U} A\right) X, \xi\right)-\left(\frac{c}{\alpha}+\alpha\right) g(A W, X)-2 c w(X),
\end{aligned}
$$

where we have used (2.4), (2.5) and (4.3), which together with (4.7) implies that

$$
\begin{aligned}
& \mu\left(\nabla_{U} A\right) \xi-\mu^{2}\left\{\left(\alpha+\frac{c}{\alpha}\right) A W+2 c W\right\} \\
& =(U \mu) A \xi+\frac{c}{\alpha}\left((W \alpha) U-\lambda \mu^{2} W-\mu \rho A \xi\right) \\
& \quad+c \alpha \mu \xi-\alpha \mu^{2} A W .
\end{aligned}
$$

If we apply by $\phi$ to this and make use of (3.5) and (4.9), then we obtain

$$
\nabla_{U} U=-\frac{c}{\alpha}(W \alpha) W+\delta U
$$

for some function $\delta$ on $\Omega$.

On the other hand, differentiating (4.3) covariantly, 
and using itself again, we find

$$
-\frac{c}{\alpha}(X \alpha) U+\alpha\left(\nabla_{X} A\right) U+\alpha A \nabla_{X} U+c \nabla_{X} U=0,
$$

which together with (2.4) and (2.5) gives

$$
\begin{aligned}
& \frac{c}{\alpha}((Y \alpha) u(X)-(X \alpha) u(Y)) \\
& +c \alpha \mu(\eta(X) w(Y)-\eta(Y) w(X)) \\
& +\alpha\left(g\left(A \nabla_{X} U, Y\right)-g\left(A \nabla_{Y} U, X\right)\right)+c d u(X, Y)=0,
\end{aligned}
$$

where $d u$ is the exterior derivative of a 1 -form $u$ is given by

$$
d u(X, Y)=X(u(Y))-Y(u(X))-u([X, Y])
$$

Putting $X=\xi$ in (4.11) and taking account of (2.7) and (2.10), we get

$$
\begin{aligned}
& \alpha \mu g\left(W, \nabla_{Y} U\right) \\
& =-\frac{c}{\alpha}(\xi \alpha) u(Y)+c \alpha \mu w(Y)+\mu\left(\alpha^{2}+c\right) g(A W, Y) \\
& +g\left(\alpha A \nabla_{\xi} U+c \nabla_{\xi} U, Y\right),
\end{aligned}
$$

or putting $Y=U$ and making use of (4.3),

$$
\alpha^{2} g\left(\nabla_{U} U, W\right)=-c \mu(\xi \alpha) \text {. }
$$

From this and (4.8) it follows that

$$
\alpha(W \alpha)=\mu(\xi \alpha)
$$

because $U$ is orthogonal to $W$. Comparing this with (4.10), we have

$$
\nabla_{U} U=-\frac{c}{\alpha^{2}} \mu(\xi \alpha) W+\delta U,
$$

which together with (4.3) implies that

$$
\alpha A \nabla_{U} U+c \nabla_{U} U=-\frac{c}{\alpha} \mu(\xi \alpha)\left(A W+\frac{c}{\alpha} W\right) .
$$

By virtue of (2.7), (3.5) and (4.2), we can write this as

$$
\alpha A \nabla_{U} U+c \nabla_{U} U=-\frac{c}{\alpha^{2}} \mu^{2}(\xi \alpha) A \xi .
$$

If we put $X=U$ in (4.11) and take account of the last equation, then we obtain

$$
\mu^{2}(Y \alpha)-(U \alpha) u(Y)-\frac{1}{\alpha} g\left(\mu^{2}(\xi \alpha) A \xi, Y\right)=0 .
$$

Therefore we have

$$
\alpha \nabla \alpha=\frac{\alpha(U \alpha)}{\mu^{2}} U+(\xi \alpha) A \xi .
$$

Using (4.2) and (4.3), we can write (3.14) as $\frac{1}{2}(\alpha \nabla \rho-\rho \nabla \alpha)=c\left(2-\frac{2 \rho}{\alpha}-\frac{3 \rho}{\alpha^{2}}\right) U+(\xi \rho) A \xi-A \nabla \alpha$, which together with (4.4) implies that

$$
A \nabla \alpha+3 c\left(\frac{c}{\alpha^{2}}-1\right) U=(\xi \alpha) A \xi+(W \alpha) A W .
$$

If we take the inner product $U$ to this, and make use of (4.3), we deduce that

$$
\alpha(U \alpha)=3\left(c-\alpha^{2}\right) \mu^{2}
$$

Thus, (4.13) reformed as

$$
\alpha \nabla \alpha=3\left(c-\alpha^{2}\right) U+\theta A \xi,
$$

where we have put $\theta=\xi \alpha$.

Now, we are going to prove that $\theta=0$ on $\Omega$. For this, the last equation is rewritten as

$$
\frac{1}{2} Y \alpha^{2}=3\left(c-\alpha^{2}\right) u(Y)+\theta \eta(A Y)
$$

Differentiating this with respect to a vector field $X$ again, and taking the skew-symmetric parts with respect to $X$ and $Y$, then we eventually have

$$
\begin{aligned}
& (X \theta) \eta(A Y)-(Y \theta) \eta(A X)+3\left(c-\alpha^{2}\right) d u(X, Y) \\
& =\theta\{6(\eta(A X) u(Y)-\eta(A Y) u(X)) \\
& \quad-2 g(A \phi A X, Y)+2 c g(\phi X, Y)\} .
\end{aligned}
$$

Putting $Y=\xi$ in this, we find

$$
\begin{aligned}
& \alpha(X \theta)-(\xi \theta) \eta(A X)+3\left(c-\alpha^{2}\right) d u(X, \xi) \\
& =2 \theta(u(A X)-3 \alpha u(X)) .
\end{aligned}
$$

By the way, we see, using (2.10) and (2.13), that $-d u(X, \xi)=g(\mu A W+3 \phi A U+\alpha A \xi-\beta \xi+\phi \nabla \alpha, X)$, which together with (2.7), (2.8), (3.5) and (4.3) gives

$$
-d u(X, \xi)=g\left(\mu\left(\rho+\frac{3 c}{\alpha}\right) W+\phi \nabla \alpha, X\right) .
$$

Thus it follows, using (2.5) and (4.14), that

$$
d u(X, \xi)=-g\left(\mu(\rho+3 \alpha) W+\frac{\theta}{\alpha} U, X\right) .
$$

Substituting this into (4.16), we find

$$
\begin{aligned}
& \alpha \nabla \theta-(\xi \theta) A \xi \\
& =3 \mu\left(c-\alpha^{2}\right)(\rho+3 \alpha) W+\theta\left(\frac{c}{\alpha}-9 \alpha\right) U,
\end{aligned}
$$

where we have used (4.3). Comparing this to (4.15), we get

$$
\begin{aligned}
& \left(c-\alpha^{2}\right) \\
& \{\mu(\rho+3 \alpha)(w(X) \eta(Y)-w(Y) \eta(X))+d u(X, Y)\} \\
& =\theta\left(\frac{c}{3 \alpha^{2}}-1\right)(\eta(A X) u(Y)-\eta(A Y) u(X)) \\
& +\frac{2}{3} \theta(g(A \phi A X, Y)+c g(\phi X, Y)) .
\end{aligned}
$$


If we take the inner product $\xi$ to (4.4) and make use of (2.8), (4.2) and (4.12), then we obtain

$$
\alpha^{2}(\xi \rho)=(\rho \alpha+2 c) \xi \alpha .
$$

Using this, we can write (4.4) as

$$
\begin{aligned}
& \frac{1}{2} \alpha^{2}(\alpha \nabla \rho-\rho \nabla \alpha) \\
& =-c \alpha(2 \rho+\alpha) U+c(\xi \alpha)(2 A \xi-\alpha \xi),
\end{aligned}
$$

where we have used (2.7), (2.8), (3.5), (4.2) and (4.12). Combining this with (4.14), we obtain

$$
\begin{aligned}
& \frac{1}{2} \alpha^{3} \nabla \rho-\left(\frac{1}{2} \rho \alpha^{2}+2 c \alpha\right) \nabla \alpha \\
& =c\left(5 \alpha^{2}-2 \rho \alpha-6 c\right)-c \alpha(\xi \alpha) \xi,
\end{aligned}
$$

which tells us that

$$
\begin{aligned}
& \frac{1}{2} \alpha^{3}((X \rho)(Y \alpha)-(Y \rho)(X \alpha)) \\
& =c\left(5 \alpha^{2}-2 \rho \alpha-6 c\right)((Y \alpha) u(X)-(X \alpha) u(Y)) \\
& \quad-c \alpha(\xi \alpha)(\eta(X)(Y \alpha)-\eta(Y)(X \alpha)) .
\end{aligned}
$$

Using the quite same method as that used to (4.19) from (4.14), we can drive from (4.21) the following:

$$
\begin{aligned}
& \left(10 \alpha-6 \rho-\frac{24}{\alpha} c\right)((X \alpha) u(Y)-(Y \alpha) u(X)) \\
& \quad+3(\xi \alpha)(\eta(X)(Y \alpha)-\eta(Y)(X \alpha)) \\
& \quad+2 \alpha((X \rho) u(Y)-(Y \rho) u(X)) \\
& =\alpha(\eta(X)(Y \theta)-\eta(Y)(X \theta)) \\
& \quad-\alpha(\xi \alpha) g((\phi A-A \phi) X, Y) \\
& \quad+\left(5 \alpha^{2}-2 \rho \alpha-6 c\right) d u(X, Y),
\end{aligned}
$$

where we have used (2.2) and (4.22). Putting $X=\xi$ in this and using (4.14), (4.17) and (4.20), we obtain

$$
\begin{aligned}
& \left(3 \alpha+2 \rho+\frac{5}{\alpha} c\right)(\xi \alpha) U+\alpha(\nabla \theta-(\xi \theta) \xi) \\
& +\mu\left\{(\rho+3 \alpha)\left(5 \alpha^{2}-2 \rho \alpha-6 c\right)-\frac{3}{\alpha}(\xi \alpha)^{2}\right\} W=0,
\end{aligned}
$$

which together with (2.7) and (4.18) implies that

$$
\begin{aligned}
& \left(6 \alpha-2 \rho-\frac{6}{\alpha} c\right)(\xi \alpha) U \\
& =\mu\left\{\xi \theta+(\rho+3 \alpha)\left(2 \alpha^{2}-2 \rho \alpha-3 c\right)-\frac{3}{\alpha}(\xi \alpha)^{2}\right\} W .
\end{aligned}
$$

Thus, it follows that

$$
\left(6 \alpha^{2}-2 \rho \alpha-6 c\right) \xi \alpha=0
$$

because $U$ and $W$ are orthogonal to each other, and hence $6 \alpha^{2}-2 \rho \alpha-6 c=0$ if $\xi \alpha \neq 0$. Differentiation gives $(6 \alpha-\rho) \nabla \alpha=\alpha \nabla \rho$ on this subset, which together with (4.20) yields $c=0$, a contradiction. Thus, $\xi \alpha=0$ on $\Omega$ is proved. Consequently we prove that $W \alpha=0$ by virtue of (4.12) and Remark 1. By (4.20) we also have $\xi \rho=0$. Therefore (4.14) and (4.21) are reduced respectively to

$$
\begin{gathered}
\alpha \nabla \alpha=3\left(c-\alpha^{2}\right) U \\
\frac{1}{2} \alpha^{3} \nabla \rho-\left(\frac{1}{2} \rho \alpha^{2}+2 c \alpha\right) \nabla \alpha=c\left(5 \alpha^{2}-2 \rho \alpha-6 c\right) U
\end{gathered}
$$

From (4.24) we have $\left(\alpha^{2}-c\right) d u(X, Y)=0$ and hence $\left(\alpha^{2}-c\right) d u(X, \xi)=0$, which together with (4.17) gives

$$
\left(\alpha^{2}-c\right)(\rho+3 \alpha)=0 .
$$

If $\alpha^{2}-c \neq 0$ on $\Omega$, and then we restrict our arguments on such a place. Then we have $\rho+3 \alpha=0$ and thus $U \rho=-3(U \alpha)$, which together with (4.5) yields $2 \rho+\alpha=0$, a contradiction because of Remark 1 . Accordingly we have $\alpha^{2}=c$ on $\Omega$ and hence $\alpha$ is constant. Thus, (4.25) becomes

$$
\frac{1}{2} \nabla \rho=-(2 \rho+\alpha) U
$$

On the other hand, using $\xi \alpha=0$ and the last equation, we can write (4.23) as

$\left(5 \alpha^{2}-2 \rho \alpha-6 c\right) d u(X, \xi)=0$ and consequently $(2 \rho+\alpha)(\rho+3 \alpha)=0$ by virtue of (4.17). Therefore we have $2 \rho+\alpha=0$, which together with (4.2) and $\alpha^{2}=c$ implies that $2 \beta=\alpha^{2}$, a contradiction. Thus, we deduce that $\Omega=\varnothing$. Accordingly we have

Lemma 1. $\Omega=\varnothing$ if it satisfies $\nabla_{\phi \nabla_{\xi} \xi} R_{\xi}=0$ and $R_{\xi} A=A R_{\xi}$.

From this we conclude that

Theorem 1. Let $M$ be a real hypersurface in $M_{n}(c), c \neq 0$. If it satisfies $\nabla_{\phi \nabla_{\xi} \xi} R_{\xi}=0$ and at the same time $R_{\xi} A=A R_{\xi}$, then $M$ is a Hopf hypersurface in $M_{n}(c)$.

\section{Real Hypersurfaces with $\operatorname{Tr} R_{\xi}=$ const}

In this section, we will continue our arguments under the same hypotheses as those stated in section 3, namely $R_{\xi} S=S R_{\xi}$ and $\nabla_{\phi \nabla_{\xi} \xi} R_{\xi}=0$ hold on $M$. Then we have

$$
h=\rho .
$$

Indeed, if not, then we have $\beta=\rho \alpha+c$ because of (3.3). Thus, (3.4) becomes $A^{2} \xi=\rho A \xi+c \xi$. So we have $R_{\xi} A=A R_{\xi}$ by virtue of the first equation of section 4 . By Lemma 1 , we verify that $\Omega=\varnothing$, a contradiction. Thus, $h=\rho$ is established on the whole space. 
Furthermore, we assume that if $\operatorname{Tr} R_{\xi}=$ const. Then we obtain from (2.14)

$$
\beta-h \alpha=\text { const. }
$$

This is equivalent to $g(S \xi, \xi)=$ const . by virtue of (2.6). Because of (3.5), we can write (3.18) as

$$
\lambda(\alpha A U+c U)=0 .
$$

Frist of all, we prove

Lemma 2. $\alpha A U+c U=0$ on $\Omega$ if $\operatorname{Tr} R_{\xi}=$ const .

Proof. If not, then we have $\lambda=0$, that is $h-\alpha=0$ with the aid of (5.1) and (5.3). And we restrict arguments on such a place. Then (3.5) becomes

$$
A W=\mu \xi
$$

Because of (2.8), (3.12) and (5.1), we can write (3.16) as

$$
\alpha A^{2} U+2\left(\mu^{2}+c\right) A U=\mu A \nabla \mu-\mu(W \alpha) A \xi .
$$

Using (2.8) and (5.4), the Equation (3.21) reformed as

$$
\mu \nabla \mu=2 c U-\alpha A U+\mu(W \alpha) \xi .
$$

Since $h=\alpha$, we see, using (5.2), that $\nabla \mu=0$. Hence, taking the inner product $\xi$ to the above equation, we have $W \alpha=0$. Thus, we verify from the last equation

$$
\alpha A U=2 c U .
$$

We also have from (5.5) $\alpha A^{2} U+2\left(\mu^{2}+c\right) A U=0$. Combining the last two equations, we obtain $\left(\mu^{2}+2 c\right) A U=0$. Thus, we see, using (5.6), that

$$
\mu^{2}+2 c=0 .
$$

On the other hand, if we take the inner product $U$ to (2.11) and make use of (2.4), (5.4), (5.6) and (5.7), then we obtain

$$
\begin{aligned}
& g\left(\nabla_{X} W, U\right) \\
& =\frac{1}{\mu} g\left(\left(\nabla_{U} A\right) \xi, X\right)-2 c w(X)+\frac{2 c}{\mu}\left(\alpha-\frac{2 c}{\alpha}\right) \eta(X) .
\end{aligned}
$$

Applying (2.11) by $\phi$ and taking account of (2.10), we also deduce that

$$
\begin{aligned}
\phi\left(\nabla_{X} A\right) \xi= & \nabla_{X} U+\mu w(A X) \xi-\phi A \phi A X \\
& -\alpha A X+\alpha g(A \xi, X) \xi .
\end{aligned}
$$

If we put $X=U$ in this and use (5.4) and (5.6), then we get

$$
\nabla_{U} U=\phi\left(\nabla_{U} A\right) \xi+2 c U .
$$

Now, differentiating (5.4) covariantly and using (2.2) and (5.7), we find

$$
\left(\nabla_{X} A\right) W+A \nabla_{X} W=\mu \phi A X .
$$

If we take the inner product $U$ to this and make use of (2.4), (5.4), (5.6) and (5.7), then we obtain

$$
\frac{2 c}{\alpha} g\left(\nabla_{X} W, U\right)=-c \mu \eta(X)-g\left(\left(\nabla_{U} A\right) W, X\right) .
$$

By the way, we have from (3.20)

$$
\mu \alpha\left(\nabla_{W} A\right) X=-4 c(g(A \xi, X) U+u(X) A \xi),
$$

where we have used $W \alpha=0, \lambda=0$, (5.6) and (5.7), which together with (2.4) gives

$$
\left(\nabla_{U} A\right) W=2 c \mu \xi-\frac{4 c \mu}{\alpha} A \xi .
$$

Substituting this into (5.10) and using (5.7), we find

$$
g\left(\nabla_{X} W, U\right)=\frac{3 c \alpha}{\mu} \eta(X)+2 \mu g(A \xi, X),
$$

which together with (5.8) implies that

$$
\left(\nabla_{U} A\right) \xi=-2 c \mu W+c\left(-3 \alpha+\frac{4 c}{\alpha}\right) \xi .
$$

Thus, it follows that $\phi\left(\nabla_{U} A\right) \xi=-2 c U$. From this and (5.9) we verify that

$$
\nabla_{U} U=0 .
$$

Differentiating (5.6) covariantly, and using itself again, we get

$$
\frac{2 c}{\alpha}(X \alpha) U+\alpha\left(\nabla_{X} A\right) U+\alpha A \nabla_{X} U=2 c \nabla_{X} U,
$$

or, using (2.4) and (2.5)

$$
\begin{aligned}
& \frac{2 c}{\alpha}(X \alpha) u(Y)+\alpha g\left(\left(\nabla_{U} A\right) X, Y\right)-c \alpha \mu \eta(X) w(Y) \\
& -2 c \alpha \mu w(X) \eta(Y)+\alpha g\left(A \nabla_{X} U, Y\right) \\
& -2 c g\left(\nabla_{X} U, Y\right)=0 .
\end{aligned}
$$

Taking skew-symmetric part with respect to $X$ and $Y$, we find

$$
\begin{aligned}
& \frac{2 c}{\alpha}((X \alpha) u(Y)-(Y \alpha) u(X)) \\
& +c \alpha \mu(\eta(X) w(Y)-\eta(Y) w(X)) \\
& +\alpha\left(g\left(A \nabla_{X} U, Y\right)-g\left(A \nabla_{Y} U, X\right)\right)-2 c d u(X, Y)=0 .
\end{aligned}
$$

If we put $Y=U$ in this and make use of (5.6) and (5.11), then we obtain

$$
\mu^{2} \nabla \alpha=(U \alpha) U
$$

which enables us to obtain $\xi \alpha=0$. Accordingly (3.14) turns out to be

$$
3 A^{2} U-2 \alpha A U-\left(\mu^{2}+c\right) U+A \nabla \alpha=0,
$$

where we have used (2.8) and $\rho=\alpha$, which together 
with (5.6) and (5.7) gives $3 c\left(4 c-\alpha^{2}\right) U+\alpha^{2} A \nabla \alpha=0$. Thus, it is seen that $\alpha(U \alpha)=3 c\left(4 c-\alpha^{2}\right)$. So we can write (5.12) as $\nabla \alpha^{2}=3\left(\alpha^{2}-4 c\right) U$. Differentiating this covariantly and taking the skew-symmtric part, we eventually have $\left(\alpha^{2}-4 c\right) d u(X, Y)=0$, which implies that $\left(\alpha^{2}-4 c\right) d u(X, \xi)=0$. This together with (5.4), (5.6) and (5.12) yields $\alpha\left(\alpha^{2}-4 c\right)=0$, Thus we have $\alpha^{2}-4 c=0$ because of Remark 1 . It is contradictory by virtue of (5.7). Consequently Lemma 2 is proved.

Using (5.1) and Lemma 2, we can write respectively (3.20) and (3.21) as

$$
\begin{aligned}
& \alpha\left(\nabla_{W} A\right) X \\
& =-(W \alpha) A X-\frac{c}{\alpha} \lambda(w(X) U+u(X) W) \\
& +g\left(\nabla \mu-\frac{c h}{\alpha \mu} U, X\right) A \xi+g(A \xi, X)\left(\nabla \mu-\frac{c h}{\alpha \mu} U\right), \\
& \mu \nabla \mu-\alpha \nabla \lambda=(W \alpha) A W-(\xi \lambda) A \xi+c\left(3+\frac{2 \lambda}{\alpha}\right) U .
\end{aligned}
$$

Replacing $X$ by $U$ in (5.13) and remembering Lemma 2, we find

$$
\alpha\left(\nabla_{W} A\right) U=(U \mu) A \xi+\frac{c}{\alpha}\left((W \alpha) U-\lambda \mu^{2} W-\mu h A \xi\right) .
$$

On the other hand, if we take the inner product (3.7) with $U$, and make use of (2.4), (2.10) and Lemma 2, then we get

$$
\begin{aligned}
& (\alpha \lambda+c) g\left(\nabla_{X} W, U\right) \\
& =\alpha g\left(\left(\nabla_{W} A\right) U, X\right)+c \alpha \mu \eta(X)-\alpha \mu^{2} g(A W, X),
\end{aligned}
$$

which connected to the last equation implies that

$$
\begin{aligned}
& (\alpha \lambda+c) g\left(\nabla_{X} W, U\right) \\
& =g\left(X,(U \mu) A \xi+\frac{c}{\alpha}\left((W \alpha) U-\lambda \mu^{2} W-\mu h A \xi\right)\right) \\
& \quad+c \alpha \mu \eta(X)-\alpha \mu^{2} g(A W, X) .
\end{aligned}
$$

Thus, it follows that

$$
(\alpha \lambda+c) g\left(\nabla_{U} W, U\right)=\frac{c}{\alpha} \mu^{2}(W \alpha) .
$$

Using the quite same method as that used to derive (4.10) from (3.7), (4.6) and (2.11), we can derive from (3.7), (5.15) and (2.11) the following:

$$
(\alpha \lambda+c) \nabla_{U} U=-\frac{c}{\alpha} \mu^{2}(W \alpha) W+\delta U
$$

where the function $\delta$ is given by

$$
\begin{aligned}
& \delta=\mu(U \mu)+(\alpha \lambda+c) \\
& \left\{2 c+\lambda\left(\frac{c}{\alpha}+\alpha\right)\right\}-\left\{\frac{c}{\alpha}(2 \lambda+\alpha)+\alpha \lambda\right\} \mu^{2} .
\end{aligned}
$$

We notice here that the following:

Remark 2. $\alpha \lambda+c \neq 0$ on $\Omega$ if $\operatorname{Tr} R_{\xi}=$ const .

In fact, if not, then $\alpha \lambda+c=0$. So we have from (5.16) $W \alpha=0$ and hence $\delta=0$ because of (5.17). Thus, (5.18) implies that $\alpha(U \mu)=2 c \lambda \mu$ on this subset. However, by putting $X=\xi$ in (5.15) we obtain $\alpha(U \mu)=\left(\alpha \mu^{2}+c \lambda\right) \mu$. Combining the last two equations, we verify that $\alpha \mu^{2}=c \lambda$, which together with $\alpha \lambda+c=0$ gives $\mu^{2}+\lambda^{2}=0$, which will produce a contradiction. Therefore $\alpha \lambda+c \neq 0$ on $\Omega$ is proved.

Because of (5.17) and Lemma 2, it is seen that

$$
\alpha A \nabla_{U} U+c \nabla_{U} U=-\frac{c}{\alpha \lambda+c} \mu^{2}(W \alpha)\left(A W+\frac{c}{\alpha} W\right)
$$

by virtue of Remark 2 .

Now, differentiating $\alpha A U+c U=0$ covariantly and using itself, we find

$$
-\frac{c}{\alpha}(X \alpha) U+\alpha\left(\nabla_{X} A\right) U+\alpha A \nabla_{X} U+c \nabla_{X} U=0,
$$

which together with (2.4) and (2.5) implies that

$$
\begin{aligned}
& \frac{c}{\alpha}((Y \alpha) u(X)-(X \alpha) u(Y)) \\
& +c \alpha \mu(\eta(X) w(Y)-\eta(Y) w(X)) \\
& +\alpha\left(g\left(A \nabla_{X} U, Y\right)-g\left(A \nabla_{Y} U, X\right)\right)+c d u(X, Y)=0 .
\end{aligned}
$$

If we put $X=\xi$ in this, and make use of (2.7) and (2.10), then we get

$$
\begin{aligned}
& \alpha \mu g\left(W, \nabla_{Y} U\right) \\
= & -\frac{c}{\alpha}(\xi \alpha) u(Y)+c \alpha \mu w(Y) \\
& +\mu\left(\alpha^{2}+c\right) g(A W, Y)+g\left(\alpha A \nabla_{\xi} U+c \nabla_{\xi} U, Y\right) .
\end{aligned}
$$

By putting $Y=U$ in this and using Lemma 2, we have $\alpha^{2} g\left(W, \nabla_{U} U\right)=-c \mu(\xi \alpha)$ and hence $\alpha^{2} g\left(\nabla_{U} W, U\right)=c \mu(\xi \alpha)$ because $U$ and $W$ are mutually orthogonal. From this and (5.16) we verify that

$$
\alpha \mu(W \alpha)=(\alpha \lambda+c)(\xi \alpha) \text {. }
$$

If we replace $X$ by $U$ in (5.20) and make use of (5.19), (5.22) and Lemma 2, we obtain

$$
\alpha \nabla \alpha=\frac{\alpha(U \alpha)}{\mu^{2}} U+\frac{\xi \alpha}{\mu}(\alpha A W+c W) .
$$

We are now going to prove the following:

Lemma 3. If $\operatorname{Tr}_{\xi}$ is constant, then we have

$$
(\alpha \lambda+c) \alpha \nabla \alpha=\alpha(W \alpha)(\alpha A W+c W)+2 f U
$$

on $\Omega$, where the function $f$ is given by 


$$
2 f=2 \alpha^{3} \lambda+4 c \alpha \lambda+2 c \alpha^{2}+3 c^{2}-\alpha^{2} \mu^{2} .
$$

Proof. Using (2.7), (2.8) and Lemma 2, we can write (2.13) as

$$
\alpha \nabla_{\xi} U=\left(\alpha^{2}+3 c\right) \mu W-\alpha \mu^{2} \xi+\alpha \phi \nabla \alpha,
$$

which together with (3.5), (5.22) and (5.23) implies that

$$
\nabla_{\xi} U=\left(\alpha+\frac{3 c}{\alpha}-\frac{U \alpha}{\mu^{2}}\right) \mu W-\mu^{2} \xi+\frac{W \alpha}{\mu} U .
$$

From this and Lemma 2, we obtain

$$
\begin{aligned}
& \alpha A \nabla_{\xi} U+c \nabla_{\xi} U \\
& =\left(\alpha+\frac{3 c}{\alpha}-\frac{U \alpha}{\mu^{2}}\right) \mu(\alpha A W+c W)-\mu^{2}(\alpha A \xi+c \xi) .
\end{aligned}
$$

Using (5.12), Lemma 2 and the last equation, we have

$$
\begin{aligned}
\alpha g\left(\nabla_{X} W, U\right) \\
=\frac{c}{\alpha \mu}(\xi \alpha) u(X)+\frac{U \alpha}{\mu^{2}} g(\alpha A W+c W, X) \\
-2\left(\alpha^{2}+2 c\right) g(A W, X) \\
-c\left(2 \alpha+\frac{3 c}{\alpha}\right) w(X)+\mu g(\alpha A \xi+c \xi, X) .
\end{aligned}
$$

By virtue of this and (5.15), it is verified that

$$
\begin{aligned}
& \frac{\alpha \lambda+c}{\mu^{2}}(U \alpha)(\alpha A W+c W)-\alpha(U \mu) A \xi \\
= & -c \lambda \mu^{2} W-c(\lambda+\alpha) \mu A \xi+c \alpha^{2} \mu \xi-\alpha^{2} \mu^{2} A W \\
& +(\alpha \lambda+c) \\
& \left\{2\left(\alpha^{2}+2 c\right) A W+c\left(2 \alpha+\frac{3 c}{\alpha}\right) W-\mu(\alpha A \xi+c \xi)\right\} .
\end{aligned}
$$

Since $\xi$ and $W$ are mutually orthogonal, we see from the last equation the following:

$$
\begin{aligned}
& \frac{\alpha \lambda+c}{\mu^{2}} U \alpha=-\left(c \lambda+\alpha \mu^{2}\right)+\frac{\alpha(U \mu)}{\mu}+(\alpha \lambda+c)\left(\alpha+\frac{3 c}{\alpha}\right), \\
& \frac{(\alpha \lambda+c)^{2}}{\mu^{2}} U \alpha=\alpha \mu(U \mu)-\left(2 c \lambda+c \alpha+\lambda \alpha^{2}\right) \mu^{2} \\
& +(\alpha \lambda+c)\left\{2\left(\alpha^{2}+2 c\right) \lambda+c\left(2 \alpha+\frac{3 c}{\alpha}\right)-\alpha \mu^{2}\right\} .
\end{aligned}
$$

Eliminating $U \alpha$ from above two equations, we eventually have

$$
\left\{\alpha(U \mu)-\left(\lambda \alpha^{2}+2 c \lambda+c \alpha\right)\right\} \mu\left(\alpha \lambda+c-\mu^{2}\right)=0 .
$$

Now, suppose that $\alpha(U \mu)-\left(\lambda \alpha^{2}+2 c \lambda+c \alpha\right) \mu \neq 0$ on $\Omega$. Then we have $\alpha \lambda+c-\mu^{2}=0$, which together with (2.8) and (5.1) yields $\beta-\rho \alpha-c=0$ on this subset.
So (3.4) becomes $A^{2} \xi=\rho A \xi+c \xi$ and consequently $R_{\xi} A=A R_{\xi}$ on this set. By Lemma 1 we see that $\Omega=\varnothing$, a contradiction. Therefore we have

$$
\alpha(U \mu)=\left(\lambda \alpha^{2}+2 c \lambda+c \alpha\right) \mu
$$

with the aid of (5.27). Comparing this with (5.26), it follows that

$$
\begin{aligned}
& \alpha(\alpha \lambda+c) U \alpha \\
& =\left(2 \alpha^{3} \lambda+4 c \alpha \lambda+2 c \alpha^{2}+3 c^{2}-\alpha^{2} \mu^{2}\right) \mu^{2} .
\end{aligned}
$$

Therefore, (5.24) and (5.25) are established on $\Omega$ because of (5.22), (5.23) and (5.29). This completes the proof.

Now, differentiating (2.7) covariantly and using (2.2), we find

$$
\begin{aligned}
& \left(\nabla_{X} A\right) \xi+A \phi A X \\
& =(X \alpha) \xi+\alpha \phi A X+(X \mu) W+\mu \nabla_{X} W .
\end{aligned}
$$

Putting $X=\xi$ in this and making use of (2.12) and (3.23), we find

$$
3 A U-\alpha U-(\xi \alpha) \xi-(W \alpha) W+\nabla \alpha=\mu \nabla_{\xi} W,
$$

which together with (5.22), (5.24), (5.25) and Lemma 2 gives

$$
\mu \nabla_{\xi} W=\frac{1}{\alpha \lambda+c}\left(\alpha^{2} \lambda+c \lambda+c \alpha-\alpha \mu^{2}\right) U .
$$

By the way, if we replace $X$ by $\xi$ in (3.7) and take account of (3.11), (3.23) and Lemma 1, then we obtain

$$
\begin{aligned}
& \mu\left(A \nabla_{\xi} W-\lambda \nabla_{\xi} W\right) \\
& =\left(\mu^{2}+\frac{c}{\alpha} \lambda\right) U-\mu \nabla \mu+\mu(\xi \lambda) W+\mu(W \alpha) \xi,
\end{aligned}
$$

which connected to the last equation and Lemma 1 implies that

$$
\mu \nabla \mu=\mu(W \alpha) \xi+\mu(\xi \lambda) W+\left(\alpha \lambda+\frac{2 c \lambda}{\alpha}+c\right) U .
$$

Using this and (3.24), we can write (5.14) as

$$
\alpha \nabla \lambda=\alpha(\xi \lambda) \xi+\alpha(W \lambda) W+(\alpha \lambda-2 c) U .
$$

From (5.2) we have

$$
\nabla \beta=h(\nabla \alpha)+\alpha(\nabla h),
$$

which shows that $\xi \beta=h(\xi \alpha)+\alpha(\xi h)$. Since we have

$$
2 \mu(W \alpha)=\xi \beta-2 \alpha(\xi \alpha)
$$

because of (2.8) and (3.23), we verify, using the last equation, that $2 \mu(W \alpha)=\alpha(\xi h)+(h-2 \alpha) \xi \alpha$. By virtue of this and (5.22), it is seen that $2(\alpha \lambda+c) \xi \alpha=\alpha^{2}(\xi h)+\alpha(\lambda-\alpha) \xi \alpha$. Thus, it follows that 


$$
\alpha^{2}(\xi \lambda)=(\alpha \lambda+2 c) \xi \alpha .
$$

If we take the inner product $U$ to (5.32), then we have $\alpha(U \lambda)=(\alpha \lambda-2 c) \mu^{2}$, which together with (5.33) gives

$$
U \beta=(\alpha \lambda-2 c) \mu^{2}+(\alpha+h) U \alpha .
$$

On the other hand, we have from (2.8)

$$
U \beta=2 \mu(U \mu)+2 \alpha(U \alpha) .
$$

Combining to the last two equations, we verify that

$$
2 \mu(U \mu)=(\alpha \lambda-2 c) \mu^{2}+\lambda(U \alpha),
$$

which together with (5.28) and (5.29) implies that

$$
\alpha^{3} \lambda^{2}-3 c \alpha^{2} \lambda-c^{2} \lambda-4 c^{2} \alpha=\alpha^{2} \lambda \mu^{2} .
$$

Differentiating this with respect to $\xi$ and using (3.23) and (5.22), we find

$$
\begin{aligned}
& \left(\alpha^{2} \lambda^{2}-8 c \alpha \lambda-2 \alpha \lambda \mu^{2}-4 c^{2}\right) \xi \alpha \\
& +\left(2 \alpha^{3} \lambda-3 c \alpha^{2}-c^{2}-\alpha^{2} \mu^{2}\right) \xi \lambda=0,
\end{aligned}
$$

which together with (5.34) and (5.35) yields

$$
\left(\lambda^{2}+4 \alpha \lambda+4 c\right) \alpha(\xi \alpha)=0 \text {. }
$$

From this and (5.34), we easily see that $\xi \alpha=0$. So it is, using (5.22) and (5.34), seen that $W \alpha=0$ and $\xi \lambda=0$. Hence (3.24) becomes $W \lambda=0$. Using these facts, (5.24), (5.31) and (5.32) turn out respectively to

$$
(\alpha \lambda+c) \alpha \nabla \alpha=\left(2 \alpha^{3} \lambda+4 c \alpha \lambda+2 c \alpha^{2}+3 c^{2}-\alpha^{2} \mu^{2}\right) U,
$$

$$
\alpha \mu \nabla \mu=\left(\alpha^{2} \lambda+2 c \lambda+c \alpha\right) U
$$

and

$$
\alpha \nabla \lambda=(\alpha \lambda-2 c) U
$$

Now, we prove

Lemma 4. $\alpha \lambda=2 c$ and $2 \lambda+3 \alpha=0$ on $\Omega$ if $\operatorname{Tr} R_{\xi}=$ const .

Proof. From (5.38) we have

$$
\begin{aligned}
& (X \alpha)(Y \lambda)+\alpha X(Y \lambda) \\
& =(\lambda(X \alpha)+\alpha(X \lambda)) u(Y)+(\alpha \lambda-2 c) X(u(Y)) .
\end{aligned}
$$

If we take the skew-symmtric parts of this and make use of (5.36) and (5.38), then we obtain $(\alpha \lambda-2 c) d u(X, Y)=0$ and hence

$$
(\alpha \lambda-2 c) d u(X, \xi)=0 .
$$

In the same way we see from (5.36) that

$$
\left(\alpha^{2} \lambda+2 c \lambda+c \alpha\right) d u(X, \xi)=0 .
$$

Now, we assume $\alpha \lambda-2 c \neq 0$ on $\Omega$ and that restrict our arguments on such a place. Then we have $d u(\xi, X)=0$, that is $g\left(\nabla_{X} \xi, U\right)+g\left(\nabla_{\xi} U, X\right)=0$, which together with (2.10) and (2.13) gives

$$
3 \phi A U+\alpha A \xi-\beta \xi+\phi \nabla \alpha+\mu A W=0 .
$$

Thus, it is, using (2.7), (2.8), (3.5) and Lemma 2, seen that

$$
\alpha \nabla \alpha=\left(\alpha \lambda+\alpha^{2}+3 c\right) U
$$

where we have used the fact that $\xi \alpha=0$, which connected to (5.36) implies that $\mu^{2}=\alpha \lambda-\lambda^{2}+c$. Differentiation gives $2 \mu \nabla \mu=(\alpha-2 \lambda) \nabla \lambda+\lambda \nabla \alpha$, or using (5.37), (5.38) and (5.40)

$$
\begin{aligned}
& 2\left(\alpha^{2} \lambda+2 c \lambda+c \alpha\right) \\
& =(\alpha-2 \lambda)(\alpha \lambda-2 c)+\lambda\left(\alpha \lambda+\alpha^{2}+3 c\right) .
\end{aligned}
$$

Therefore, we obtain

$$
\alpha \lambda^{2}+4 c \alpha-3 c \lambda=0 .
$$

If we differentiate this, then we have $\left(\lambda^{2}+4 c\right) \nabla \alpha+(2 \alpha \lambda-3 c) \nabla \lambda=0$, which together with (5.38) and (5.40) gives

$$
\alpha \lambda^{3}+3 \lambda^{2} \alpha^{2}+3 c \lambda^{2}-3 c \alpha \lambda+4 c \alpha^{2}+18 c^{2}=0 .
$$

Combining this to (5.41), we obtain $\lambda^{6}+12 c \lambda^{4}+32 c^{2} \lambda^{2}+48 c^{3}=0$. This means that $\lambda$ is constant and hence $\alpha \lambda=2 c$ because of (5.38), a contradiction. Accordingly $\alpha \lambda=2 c$ is valid on $\Omega$.

In the same way we verify from (5.39) that $\alpha^{2} \lambda+2 c \lambda+c \alpha=0$, which together with $\alpha \lambda=2 c$ implies that $2 \lambda+3 \alpha=0$ on $\Omega$. This completes the proof of Lemma 4.

Putting $X=W$ in (5.30) and using (3.10), Lemma 2 and Lemma 4, we get

$$
\nabla_{W} W=0 .
$$

If we put $X=\mu W$ in (2.11) and make use of (3.5), (3.10), (5.36), (5.37) and Lemma 4, then we obtain $\phi \nabla_{W} U=0$, which together with (2.10) and (3.5) yields

$$
\nabla_{W} U=-\mu \lambda \xi \text {. }
$$

Finally we prove

Theorem 2. Let $M$ be a real hypersurface with $\operatorname{Tr}_{\xi}=$ const. in $M_{n}(c), c \neq 0$. If it satisfies $\nabla_{\phi \nabla_{\xi} \xi} R_{\xi}=0$ and at the same time $R_{\xi} S=S R_{\xi}$, then $M$ is a Hopf hypersurface, where $S$ denotes the Ricci tensor of $M$.

Proof. From Lemma 4, we see that

$$
\alpha h=\alpha^{2}+2 c, 2 h+\alpha=0 .
$$

Because of Lemma 4, we also verify that $\alpha, \mu$ and $\lambda$ are constant on $\Omega$ by virtue of (5.36)-(5.38). Using these and (5.44) we can write (5.13) as 


$$
\begin{aligned}
& \left(\nabla_{W} A\right) X= \\
& u(X)\left(\frac{c}{2 \mu} \xi+\frac{2 c}{\alpha} W\right)+\left(\frac{c}{2 \mu} \eta(X)+\frac{2 c}{\alpha} w(X)\right) U,
\end{aligned}
$$

which together with the Codazzi Equation (2.4) implies that

$$
\begin{aligned}
& \left(\nabla_{X} A\right) W \\
& =u(X)\left(\frac{5 c}{2 \mu} \xi+\frac{2 c}{\alpha} W\right)+\left(\frac{3 c}{2 \mu} \eta(X)+\frac{2 c}{\alpha} w(X)\right) U .
\end{aligned}
$$

Differentiating this covariantly and using (2.2), we find

$$
\begin{aligned}
& \left(\nabla_{Y} \nabla_{X} A\right) W+\left(\nabla_{X} A\right)\left(\nabla_{Y} W\right) \\
= & \left(\frac{2 c}{\alpha} \nabla_{Y} W+\frac{c}{2 \mu} \phi A Y\right) u(X)+\left\{\frac{2 c}{\alpha} Y(w(X))+\frac{3 c}{2 \mu}\left(g(\phi A Y, X)+g\left(\xi, \nabla_{X} Y\right)\right)\right\} U \\
& +Y(u(X))\left(\frac{2 c}{\alpha} W+\frac{5 c}{2 \mu} \xi\right)+\left(\nabla_{Y} U\right)\left(\frac{2 c}{\alpha} w(X)+\frac{3 c}{2 \mu} \eta(X)\right) .
\end{aligned}
$$

If we take the skew-symmetric part with respect to $X$ and $Y$, and using the Ricci identity, we obtain

$$
\begin{aligned}
R & (Y, X) A W-A(R(Y, X) W)+\left(\nabla_{X} A\right)\left(\nabla_{Y} W\right)-\left(\nabla_{Y} A\right)\left(\nabla_{X} W\right) \\
= & \left(\frac{2 c}{\alpha} \nabla_{Y} W+\frac{c}{2 \mu} \phi A Y\right) u(X)-\left(\frac{2 c}{\alpha} \nabla_{X} W+\frac{c}{2 \mu} \phi A X\right) u(Y) \\
& +\frac{2 c}{\alpha} d w(Y, X)+\frac{3 c}{2 \mu}\left(g(\phi A Y, X)-g(\phi A X, Y)+g\left(\xi, \nabla_{X} Y\right)-g\left(\xi, \nabla_{Y} X\right)\right) U \\
& +\left(\frac{5 c}{2 \mu} \xi+\frac{2 c}{\alpha} W\right) d u(Y, X)+\left(\nabla_{Y} U\right)\left(\frac{3 c}{2 \mu} \eta(X)+\frac{2 c}{\alpha} w(X)\right)-\left(\nabla_{X} U\right)\left(\frac{2 c}{\alpha} w(Y)+\frac{3 c}{2 \mu} \eta(Y)\right) .
\end{aligned}
$$

By putting $Y=W$ and using (2.9), (3.5), (5.42), (5.43) and (5.45), we find

$$
\begin{aligned}
& \mu R(W, X) \xi+\lambda R(W, X) W-A(R(W, X) W)=u\left(\nabla_{X} W\right)\left(\frac{c}{2 \mu} \xi+\frac{2 c}{\alpha} W\right)+\frac{c}{2 \mu} \eta\left(\nabla_{X} W\right) U \\
& -\frac{4 c}{\mu^{2}} \lambda u(X) U \frac{3 c}{2 \mu^{2}} u(X) A U+\left(\frac{5 c}{2 \mu} \xi+\frac{2 c}{\alpha} W\right) d u(Y, X)-\frac{2 c}{\alpha} \nabla_{X} U-\mu \lambda\left(\frac{2 c}{\alpha} w(X)+\frac{3 c}{2 \mu} \eta(X)\right) \xi .
\end{aligned}
$$

On the other hand, using (2.7), (3.5) and Lemma 4, we can write (5.15) as

$$
3 c g\left(\nabla_{X} W, U\right)=-\mu\left(c \lambda+\alpha \mu^{2}\right) \eta(X) .
$$

From (2.3), we have

$$
\begin{aligned}
& R(W, X) \xi=c \eta(X) W+\alpha \eta(X) A W+\mu w(X) A W-\mu A X, \\
& R(W, X) W=c(w(X) W-X)-\frac{3 c}{\mu^{2}} u(X) U+g(X, A W) A W-\lambda A X,
\end{aligned}
$$

which together with (2.8), (3.6) and (5.44) implies that

$$
A(R(W, X) W)=c(w(X) A W-A X)-\frac{3 c}{\mu^{2}} u(X) A U+g(X, A W) A^{2} W-\lambda A^{2} X .
$$

Substituting above four equations into (5.46), we find

$$
\begin{aligned}
& -\lambda A^{2} X+\left(\mu^{2}+\lambda^{2}-c\right) A X+c \lambda X-\frac{2 c}{\alpha} \nabla_{X} U+\frac{15 c^{2}}{\mu^{2}} u(X) U \\
& +c \mu(\eta(X) W-w(X) \xi)+\mu \eta(A X) A W-\mu w(A X) A \xi+\frac{\mu}{3 c}\left(c \lambda+\alpha \mu^{2}\right) \eta(X)\left(\frac{c}{2 \mu} \xi+\frac{2 c}{\alpha} W\right) \\
& =-\left(\frac{5 c}{2 \mu} \xi-\frac{2 c}{\alpha} W\right) d u(W, X)-\mu \lambda\left(\frac{2 c}{\alpha} w(X)+\frac{3 c}{2 \mu} \eta(X)\right) \xi .
\end{aligned}
$$


Since we have $g(U, U)=\mu^{2}$ from (2.5), if we take the inner product $\alpha U$ to this, then we obtain

$$
-\alpha \lambda A U^{2}+\alpha\left(\mu^{2}+\lambda^{2}-c\right) A U+17 c^{2} U=0,
$$

which together with Lemma 2 and Lemma 4 implies that

$$
\alpha^{2}\left(\mu^{2}+\lambda^{2}\right)+26 c^{2}=0
$$

which will produce a contradiction. This completes the proof.

\section{REFERENCES}

[1] T. E. Cecil and P. J. Ryan, "Focal Sets and Real Hypersurfaces in Complex Projective Space,” Transactions of the American Mathematical Society, Vol. 269, No. 2, 1982, pp. 481-499.

[2] R. Takagi, "On Homogeneous Real Hypersurfaces in a Complex Projective Space,” Osaka Journal of Mathematics, Vol. 19, 1973, pp. 495-506.

[3] R. Takagi, "Real Hypersurfaces in a Complex Projective Space with Constant Principal Curvatures I, II,” Journal of the Mathematical Society of Japan, Vol. 15, No. 43-53, 1975, pp. 507-516.

[4] M. Kimura, "Real Hypersurfaces and Complex Submanifolds in Complex Projective Space," Transactions of the American Mathematical Society, Vol. 296, No. 1, 1986, pp. 137-149.

[5] J. Berndt, "Real Hypersurfaces with Constant Principal Curvatures in Complex Hyperblic Spaces," Journal für die Reine und Angewandte Mathematik, Vol. 395, 1989, pp. 132-141.

[6] J. T. Cho and U-H. Ki, "Jacobi Operators on Real Hy- persurfaces of a Complex Projective Space," Tsukuba Journal of Mathematics, Vol. 22, 1988, pp. 145-156.

[7] J. T. Cho and U-H. Ki, "Real Hypersurfaces in Complex Space Forms with Reeb Flow Symmetric Jacobi Operator," Canadian Mathematical Bulletin, Vol. 51, No. 3, 2008, pp. 359-371.

[8] U-H. Ki, H. Kurihara, S. Nagai and R. Takagi, "Characterizations of Real Hypersurfaces of Type A in a Complex Space Form in Terms of the Structure Jacobi Operator," Mathematics Journal of Toyama University, Vol. 32, 2009, pp. 5-23.

[9] J. D. Pérez, F. G. Santos and Y. J. Suh, "Real Hypersurfaces in Nonflat Complex Space Forms with Commuting Structure Jacobi Operator," Houston Journal of Mathematics, Vol. 33, 2007, pp. 1005-1009.

[10] M. Ortega, J. D. Pérez and F. G. Santos, "Non-Existence of Real Hypersurfaces with Parallel Structure Jacobi Operator in Nonflat Complex Space Forms,” Rocky Mountain Journal of Mathematics, Vol. 36, No. 5, 2006, pp. 1603-1613.

[11] J. D. Pérez, F. G. Santos and Y. J. Suh, "Real Hypersurfaces in Complex Projective Spaces Whose Structure Jacobi Operator is D-Parallel," Bulletin of the Belgian Mathematical Society Simon Stevin, Vol. 13, No. 3, 2006, pp. 459-469.

[12] U-H. Ki, H. Kurihara and R. Takagi, “Jacobi Operators along the Structure Flow on Real Hypersurfaces in a Nonflat Complex Space Form,” Mathematics Journal of Toyama University, Vol. 33, 2009, pp. 39-56.

[13] U-H. Ki and H. Kurihara, "Real Hypersurfaces and $\xi$ Parallel Structure Jacobi Operators in Complex Space Forms," Journal of Korean Academy Sciences, Sciences Series, Vol. 48, 2009, pp. 53-78. 